\title{
Deep learning for cephalometric landmark detection: systematic review and meta-analysis
}

\author{
Falk Schwendicke ${ }^{1,2}$ (D) Akhilanand Chaurasia ${ }^{2,3} \cdot$ Lubaina Arsiwala $^{1} \cdot$ Jae-Hong Lee $^{2,4} \cdot$ Karim Elhennawy $^{5}$. \\ Paul-Georg Jost-Brinkmann ${ }^{5}$ • Flavio Demarco ${ }^{6} \cdot$ Joachim Krois $^{1,2}$
}

Received: 26 February 2021 / Accepted: 14 May 2021 / Published online: 27 May 2021

(C) The Author(s) 2021

\begin{abstract}
Objectives Deep learning (DL) has been increasingly employed for automated landmark detection, e.g., for cephalometric purposes. We performed a systematic review and meta-analysis to assess the accuracy and underlying evidence for DL for cephalometric landmark detection on 2-D and 3-D radiographs.

Methods Diagnostic accuracy studies published in 2015-2020 in Medline/Embase/IEEE/arXiv and employing DL for cephalometric landmark detection were identified and extracted by two independent reviewers. Random-effects meta-analysis, subgroup, and meta-regression were performed, and study quality was assessed using QUADAS-2. The review was registered (PROSPERO no. 227498).

Data From 321 identified records, 19 studies (published 2017-2020), all employing convolutional neural networks, mainly on 2D lateral radiographs $(n=15)$, using data from publicly available datasets $(n=12)$ and testing the detection of a mean of 30 (SD: 25 ; range.: 7-93) landmarks, were included. The reference test was established by two experts $(n=11), 1$ expert ( $n=4), 3$ experts $(\mathrm{n}=3)$, and a set of annotators $(\mathrm{n}=1)$. Risk of bias was high, and applicability concerns were detected for most studies, mainly regarding the data selection and reference test conduct. Landmark prediction error centered around a 2-mm error threshold (mean; 95\% confidence interval: $(-0.581 ; 95 \mathrm{CI}:-1.264$ to $0.102 \mathrm{~mm})$ ). The proportion of landmarks detected within this $2-\mathrm{mm}$ threshold was 0.799 ( 0.770 to 0.824$)$.

Conclusions DL shows relatively high accuracy for detecting landmarks on cephalometric imagery. The overall body of evidence is consistent but suffers from high risk of bias. Demonstrating robustness and generalizability of DL for landmark detection is needed.

Clinical significance Existing DL models show consistent and largely high accuracy for automated detection of cephalometric landmarks. The majority of studies so far focused on 2-D imagery; data on 3-D imagery are sparse, but promising. Future studies should focus on demonstrating generalizability, robustness, and clinical usefulness of DL for this objective.
\end{abstract}

Keywords Artificial intelligence $\cdot$ Convolutional neural networks $\cdot$ Evidence-based medicine $\cdot$ Meta-analysis $\cdot$ Orthodontics . Systematic review

Charité - Universitätsmedizin Berlin is a corporate member of Freie Universität Berlin and Humboldt-Universität zu Berlin.

Falk Schwendicke

falk.schwendicke@charite.de

1 Department of Oral Diagnostics, Digital Health and Health Services Research, Charité - Universitätsmedizin Berlin, Berlin, Germany

2 Topic Group Dental Diagnostics and Digital Dentistry, ITU/WHO Focus Group AI on Health, Berlin, Germany

3 Department of Oral Medicine and Radiology, King George's Medical University, Lucknow, India
4 Department of Periodontology, Daejeon Dental Hospital, Institute of Wonkwang Dental Research, Wonkwang University College of Dentistry, Daejeon, Korea

5 Department of Orthodontics, Dentofacial Orthopedics and Pedodontics, Charité - Universitätsmedizin Berlin, Berlin, Germany

6 Post-Graduate Program in Epidemiology, Federal University of Pelotas, Pelotas, Brazil 


\section{Introduction}

Medical applications using artificial intelligence (AI) are increasingly common; one of the most prolific fields in this regard is computer vision, i.e., AI-based image analysis. Deep learning (DL), a subfield of machine learning, especially DL using convolutional neural networks (CNNs) has been demonstrated to be highly suitable for computer vision. One of the most common strategies in machine learning is supervised learning, where an algorithm is exposed to pairs of data and data labels (e.g., for computer vision, an image, and the corresponding image label). During the model "training" phase, these data pairs are repeatedly shown to the algorithm by which the DL model (specifically the model weights) is iteratively optimized to minimize the error in the model predictions. A well-trained DL model learnt to represent the (nonlinear) statistical structure of the input data and its relation to the given label [1] and is eventually capable to predict a label on new, i.e., unseen data (images).

A range of relevant aspects when training and testing DL models (e.g., CNNs) for medical applications have been identified [2-4]: (1) The representativeness of the training and test datasets needs to be ensured if generalizability of the model is expected. (2) Labelling of images is complex, as there is seldom one hard "gold standard" (e.g., histological assessment) available; more often, multiple human experts label the same image, and a range of options to unify these "fuzzy" labels have been used [5]. Different strategies to establish a reliable gold standard are available, e.g., majority voting schemes. (3) The value of any model should be demonstrated, for example, by presenting its performance against that of the current standard of care (e.g., individual healthcare providers) on a separate test dataset. Presenting the performance of a model on the same dataset it learnt from will yield highly inflated performance metrics [6].

Cephalometric radiographs are taken by orthodontists to quantitatively evaluate the skeletal relationship between the cranial base and the maxilla or mandible, the relationship between maxilla and mandible, and the dentoalveolar relationship. They also serve for determining the growth pattern through quantitative and qualitative evaluations and superimposition of serial radiographs [7]. Moreover, cephalograms are required for planning orthognathic surgery $[8,9]$. A key task on such cephalometric 2-D radiographs or 3-D CT or cone beam CT (CBCT) images is landmark detection. While the value of cephalometric analysis and the definition of landmarks remains an issue of debate [10], automating this task has been identified as useful, particularly as landmarking is laborsome, requiring the time of experienced (and expensive) experts $[11,12]$. Automated landmark detection for cephalometric analysis has been in the focus for decades, while DL has been demonstrated to possibly exceed less advanced (e.g., knowledge-based or atlas-based) systems [13]. Moreover,
DL-based cephalometric software applications from different companies (e.g., CellmatIQ, Hamburg, Germany; ORCA AI, Herzliya, Israel; WebCeph, Gyeonggi-do, Korea) are by now available to orthodontists worldwide.

The number of studies involving DL for landmark detection on 2-D and 3-D cephalometric imagery is increasing rapidly, while it remains uncertain how robust and consistent the emerging body of evidence is. Moreover, it is unclear if the accuracy of DL or the quality of the studies is improving over time, or if there are differences in accuracy on 2-D versus 3-D imagery. The present systematic review and meta-analysis evaluated studies employing DL for landmark detection on cephalometric 2-D or 3-D radiographs. Our research question was as follows: What is the accuracy of DL for detecting landmarks on cephalometric radiographs?

\section{Materials and methods}

Reporting of this review and meta-analysis followed the PRISMA checklist [14]. The study protocol was registered after the initial screening stage (PROSPERO registry no. 227498). Our PICO question was as follows: In 2- or 3-D radiographic imagery suitable for cephalometric landmark detection (participants), comparing DL (intervention) versus conventional landmarking of individual experts or against a gold standard (e.g., of multiple experts) (control), what is the accuracy (outcome)?

\section{Eligibility criteria}

The following selection criteria were applied: (1) diagnostic accuracy studies employing DL, e.g., CNNs; (2) trained and tested on 2-D- or 3-D cephalometric imagery like 2-D lateral or frontal radiographs or 3-D CT or CBCT, with minimum 5 relevant landmarks to be detected and sufficient detail to extract information on the train and test dataset sizes; (3) reporting their outcome as the mean deviation from a $2-\mathrm{mm}$ prediction error threshold (e.g., studies reporting their accuracy to be below or above this threshold) (including mean and variance) or the proportion of landmarks correctly predicted within this 2-mm prediction error threshold; and (4) published 2015-2020, as we did not expect DL studies in this field to be published before that (mainly as DL was not available much earlier and the first applications in medicine evolved since 2015), in English. Only studies fulfilling all of the abovedescribed criteria were included. Studies on nonradiographic data, cephalometrically irrelevant landmarks (e.g., those of the brain), or using non-deep learning methods (e.g., knowledge- or atlas-based or involving shallow machine learning) were excluded. 


\section{Information sources and search}

We systematically screened four electronic databases (Medline via PubMed, Embase via Ovid, IEEE Xplore, arXiv) for studies published up January 2015 to December 2020. Medline and Embase are widely used and partially complementary medical databases. IEEE (Institute of Electrical and Electronics Engineers) Xplore is a library for articles, proceedings, and standards in physics, computer science, engineering, and related fields, indexing 200+ journals and 3+ million conference papers. arXiv is an archive of electronic preprints for research articles of scientific topics such as physics, mathematics, computer science, and statistics. Archived articles may be published later in more traditional journals, and while arXiv is not peer reviewed, there are moderators who review the submissions [15]. The search was overall designed to account for different publication cultures across disciplines.

A two-pronged search strategy, combining the technique of interest (AI, CNN, DL, etc.) and the diagnostic target (landmark detection, cephalometry, orthodontics), was applied. The search sequence was adapted for each database/repository, an example for Medline can be found in Fig. 1. Reviews or editorials were excluded, and cross-referencing from bibliographies was performed.

\section{Screening}

Screening of titles or abstracts was independently performed by two reviewers (FS, AC). Any disagreement was resolved by discussion. All papers which were found to be potentially eligible were assessed in full text against the inclusion criteria. Inclusion and exclusion were decided by two reviewers in consensus (FS, AC).

\section{Data collection, items, and preprocessing}

A pretested spreadsheet was used to collect data. Study characteristics, including country, year of publication, imagery (2D lateral or frontal radiographs or 3-D imagery), dataset source, size and partitions (training, test), characteristics/ architecture of the DL strategy used, number of landmarks detected, reference test and its unification in case of multiple annotators, comparators (current standard of care, e.g., clinicians), accuracy metrics, and findings, were extracted by two reviewers (FS, AC). If one study reported on several models or test datasets, these were extracted.

\section{Quality assessment}

Risk of bias was assessed using the QUADAS-2 tool [16], accounting for bias in the data selection (no inappropriate exclusions, no case-control design, random or consecutive inclusion), index test (assessment blinded for and independent of reference test), reference test (valid reference test, assessment independent from index test), flow and timing (sufficient time between index and reference, all datapoints included in analysis), as well as applicability concerns for the data (data match review question), index test (test, conduct, and interpretation match review question), and reference test (the way landmarks were established matches review question). Risk of bias was assessed independently by two reviewers, who discussed their findings in case of disagreement to come to a consensus. We do not provide further guidance as to the certainty of the evidence (e.g., using any kind of grading), but provide descriptive statistics of the individual and overall risk of bias together with meta-analytic estimates.

\section{Summary measures and data synthesis}

The criteria for a study to be included into meta-analysis was that it reported one of our two accuracy outcomes, the deviation from a 2-mm prediction error threshold (in $\mathrm{mm}$ ) or the proportion of landmarks correctly predicted within this $2-\mathrm{mm}$ prediction error threshold (reported as mean and measures of variance and/or sample size, allowing weighing of the study estimates). Our summary measures were the mean deviation from the 2-mm threshold (in $\mathrm{mm}$; only one study reported this in another metric, namely pixels, and was therefore excluded) or the proportion of landmarks predicted within this 2-mm threshold, both with their $95 \%$ confidence intervals (CI). Heterogeneity was assessed using Cochrane's Q and I ${ }^{2}$ statistics [17]. Random-effects models were used for meta-analysis; the statistical package metaphor [18], implemented in OpenMetaAnalyst [19], was employed. To allow weighting of studies for the synthesis of proportions, we recalculated the number of true predictions (within the 2-mm threshold) in the overall test dataset, accounting for its size and the number of landmarks predicted in each study. If studies reported on multiple test datasets, we handled them as independent units, accounting for this multiplicity of accuracy data. To explore reasons for heterogeneity, we performed subgroup analyses and mixed-effect meta-regression. For the latter, the unrestricted maximum likelihood method was used.

\section{Results}

\section{Study selection and characteristics}

From 321 identified studies, 40 were screened in full texts, and 19 studies were eventually included in our review and metaanalysis (Fig. 1). The 21 excluded studies, with reasons for exclusion, can be found in Table S1. The included studies (Table 1) were published between 2017 and 2020 (median: 2020) and stemmed from nine countries (Korea, 7 studies; 
Fig. 1 Flowchart of the search

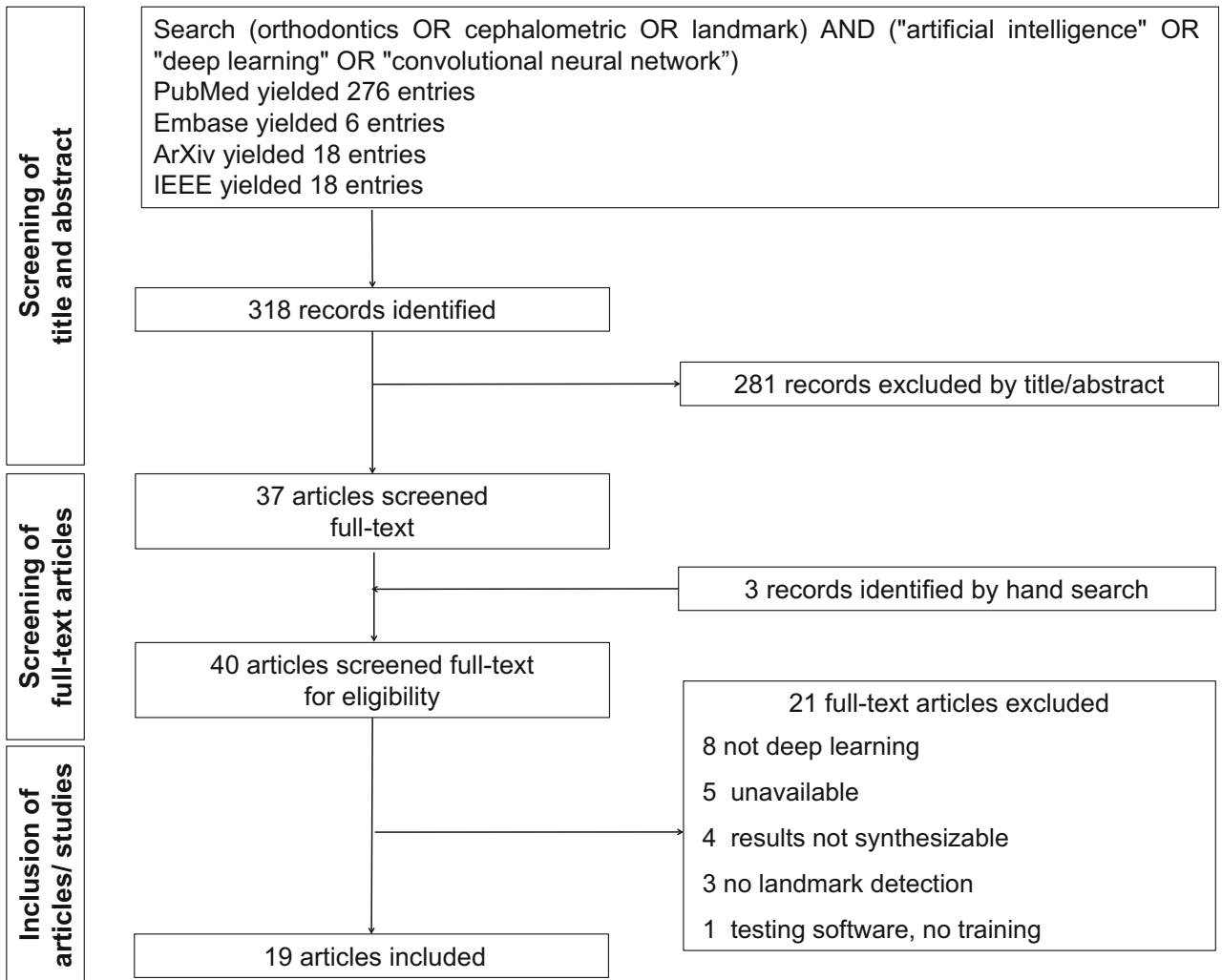

China, 4 studies; Japan, 2 studies; all six remaining countries, 1 study). Fifteen studies focused on the analysis of 2-D radiographs, four on 3-D radiographs. Eleven studies used publicly available data from the IEEE 2015 grand challenge [39]; one of these also used own data. A second publicly available dataset (CQ 500; http://headctstudy.qure.ai/dataset) was used, together with own data, by one study. Seven studies used own data; one study did not report on the dataset in detail.

All studies employed CNNs, with VGG-19 $(n=2)$ and YOLO V3 ( $n=2)$, Resnet50 $(n=2)$ and ResNet34 ( $n=2)$ being the most frequent architectures. The studies tested the detection of a mean of 30 (SD: 25; range: 7-93) landmarks. The size of the training dataset was 479 in mean $(150 ; 20-1875)$; the size of the test dataset was $128(83 ; 4-283)$.

The reference test in the training dataset was established by two experts in 11 studies, 1 expert in four studies, and 3 experts in three studies; one study used students to label the landmarks and had these corrected by experts. Eight studies used the average to come to a unified label when having more than one annotator, one used a consensus process, one used a majority voting scheme, and five studies did not report on that; for the studies with only 1 annotator, this was not relevant. The reference test in the test dataset was by large established similarly ( 2 experts: $n=13 ; 1$ expert: $n=4 ; 3$ experts: $n=1$; corrected students: $n=1$ ). Notably, many studies employed multiple test datasets, mainly as the IEEE 2015 Grand Challenge included two test datasets, one with 150 and one with 100 cephalometric images.

\section{Risk of bias and applicability concerns}

Risk of bias was assessed in four domains and found high for most studies regarding the data selection $(n=16)$, reference test $(n=18)$, but not index test $(n=7)$ or flow and timing $(n=1)$. Applicability concerns were present for most studies toward the data selection $(n=16)$, reference test $(n=18)$, but not index test $(n=8)$. A detailed assessment of risk of bias and applicability concerns can be found in Table 2 .

\section{Meta-analysis}

Two meta-analyses were performed, one synthesizing the mean deviation from a 2-mm prediction error threshold (in $\mathrm{mm}$ ) (Fig. 2) and one on the proportion of landmarks detected within this 2-mm threshold (Fig. 3). One study [33] reported the mean deviation, but not in mm but pixel. As this study would have introduced additional heterogeneity given the different outcome measure, it was not included in the first metaanalysis (on the mean deviation from the 2-mm threshold), but we could include it in the second meta-analysis (on the proportion of landmarks lying within the 2-mm threshold).

Regarding the mean deviation from a $2-\mathrm{mm}$ prediction error, an overall number of 10 studies contributed 15 estimates (three studies had tested the DL model on 2 test datasets, one study on 3 test datasets). Of these, 8 studies (13 estimates) 


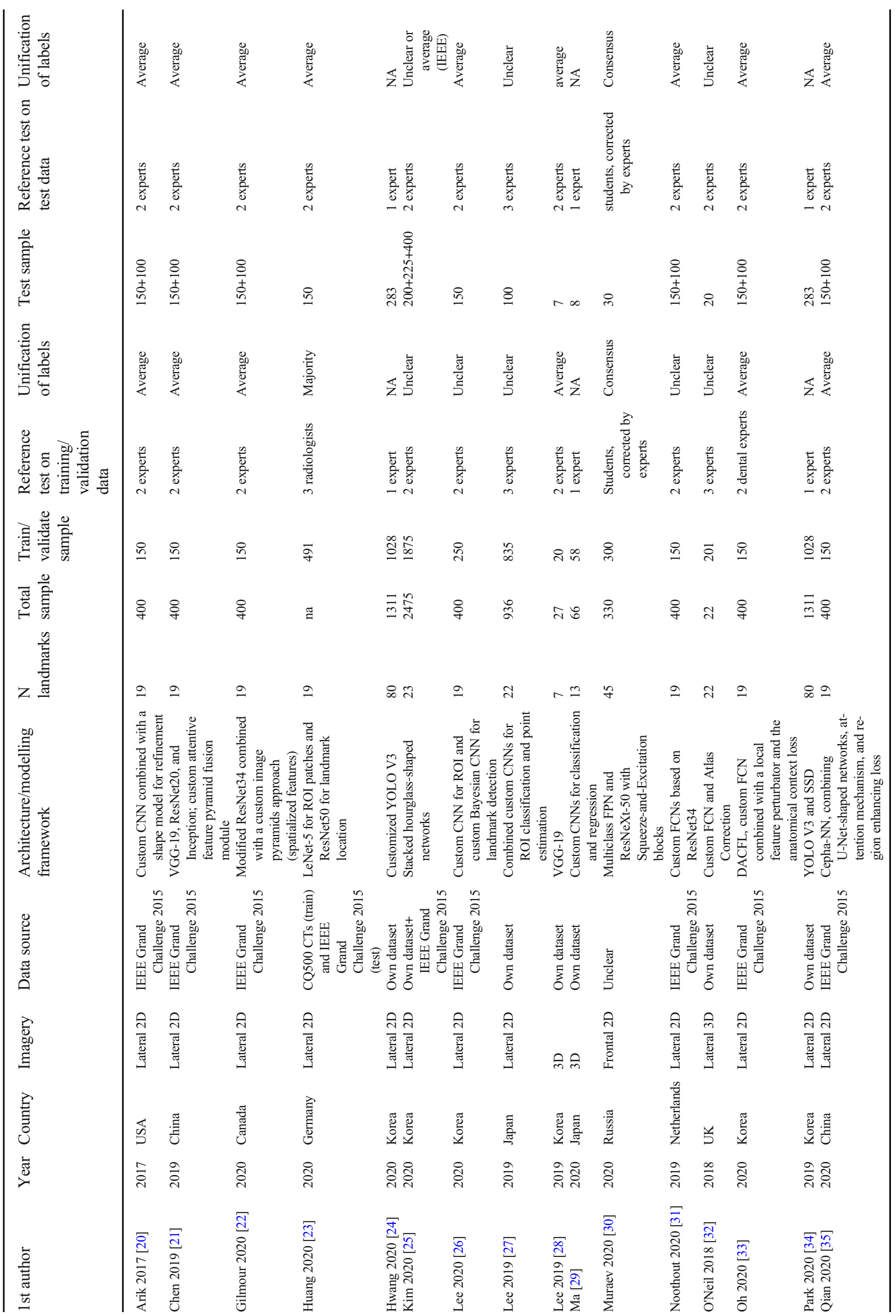




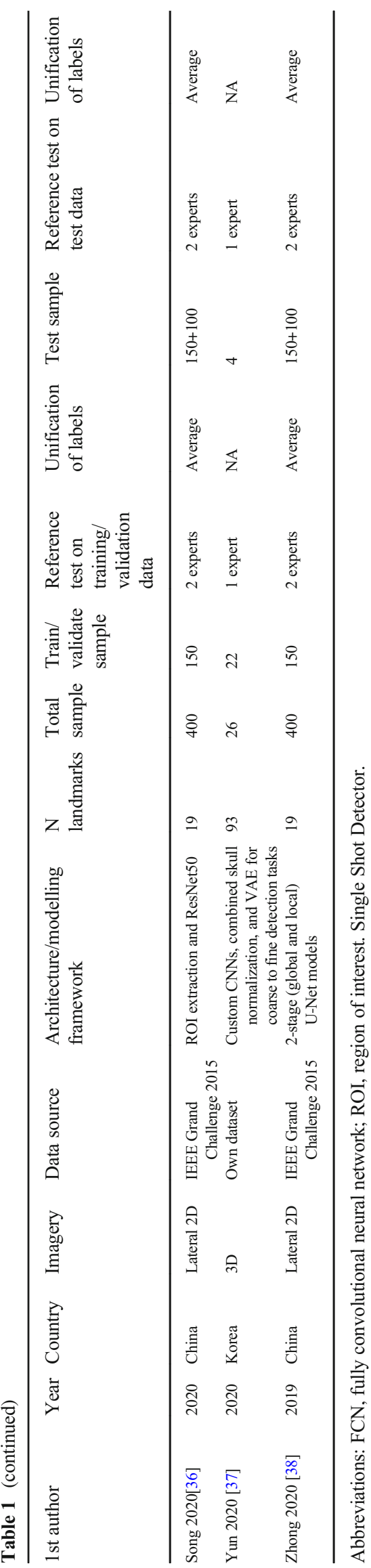

reported on 2-D radiographs, 2 on 3-D radiographs. Pooling revealed moderate heterogeneity $\left(\mathrm{I}^{2}=47 \%\right.$; $\left.\mathrm{P}=0.05\right)$, mainly due to differences between 2- and 3-D imagery. On 2-D imagery, predictions were largely below the 2-mm threshold (0.581 ; $95 \mathrm{CI}:-1264$ to $0.102 \mathrm{~mm}$ ), on 3-D imagery above the threshold ( $2.435 ;-0.038$ to $4.908 \mathrm{~mm}$ ). Overall, only 3 studies (1 on 2-D, 2 on 3-D images) had a mean deviation exceeding the 2-mm threshold (Fig. 2). The pooled deviation from the 2$\mathrm{mm}$ error was 0.054 ( -0.833 to 0.942$)$. Meta-regression revealed no significant association between the mean deviation and the year of publication $(\mathrm{P}=0.494)$.

Regarding the proportion of landmarks detected with the 2$\mathrm{mm}$ threshold, 12 studies with 22 estimates were included (20 estimates were for 2-D radiographs, 2 for 3-D radiographs); heterogeneity was high $\left(\mathrm{I}^{2}=99 \% ; \mathrm{P}<0.001\right)$. The overall proportion was 0.799 ( 0.770 to 0.824 ); the proportion was lower for 2-D $(0.792 ; 0.762$ to 0.821$)$ than $3-\mathrm{D}(0.870 ; 0.792$ to 0.948 ) imagery. Meta-regression on the year of publication did not reveal a significant association $(\mathrm{P}=0.916)$.

\section{Discussion}

In contrast to most other dental radiographs, cephalograms are not only diagnosed qualitatively but also quantitatively through angular and linear measurements often in relation to reference planes (considered as stable structures) [40]. Most quantitative analyses are based on the identification of reference points which are either skeletal landmarks, e.g., the anterior nasal spine, virtual points such as the sella (middle of sella turcica), or constructed points like the gonion (crossing point between two lines) [8]. To yield meaningful results, the precise identification of the landmarks is crucial [41]. The present review evaluated studies using DL for landmark detection on 2-D or 3-D cephalometric imagery. Based on 19 included studies, we found DL accurate for this purpose; the majority of studies did not exceed a 2-mm prediction error threshold in mean, and the mean proportion of landmarks detected within this $2-\mathrm{mm}$ threshold was $80 \%$. The findings were largely consistent across studies; the most notable difference in accuracy was found between 2-D and 3-D images. However, direction of this difference was not consistent between our two outcomes - for the mean deviation from a 2$\mathrm{mm}$ prediction error, 3-D images showed higher deviations, while for the proportion of predictions below this 2-mm threshold, 3-D images showed a higher proportion. Data on 3-D was generally sparse. The majority of studies showed high risk of bias and applicability concerns. This needs highlighting, as a number of software tools are by now already on the market - often with unclear scientific underpinning. The finding of high risk of bias and concerns in the current body of evidence is worrisome in this regard. 
Table 2 Risk of bias and applicability concerns

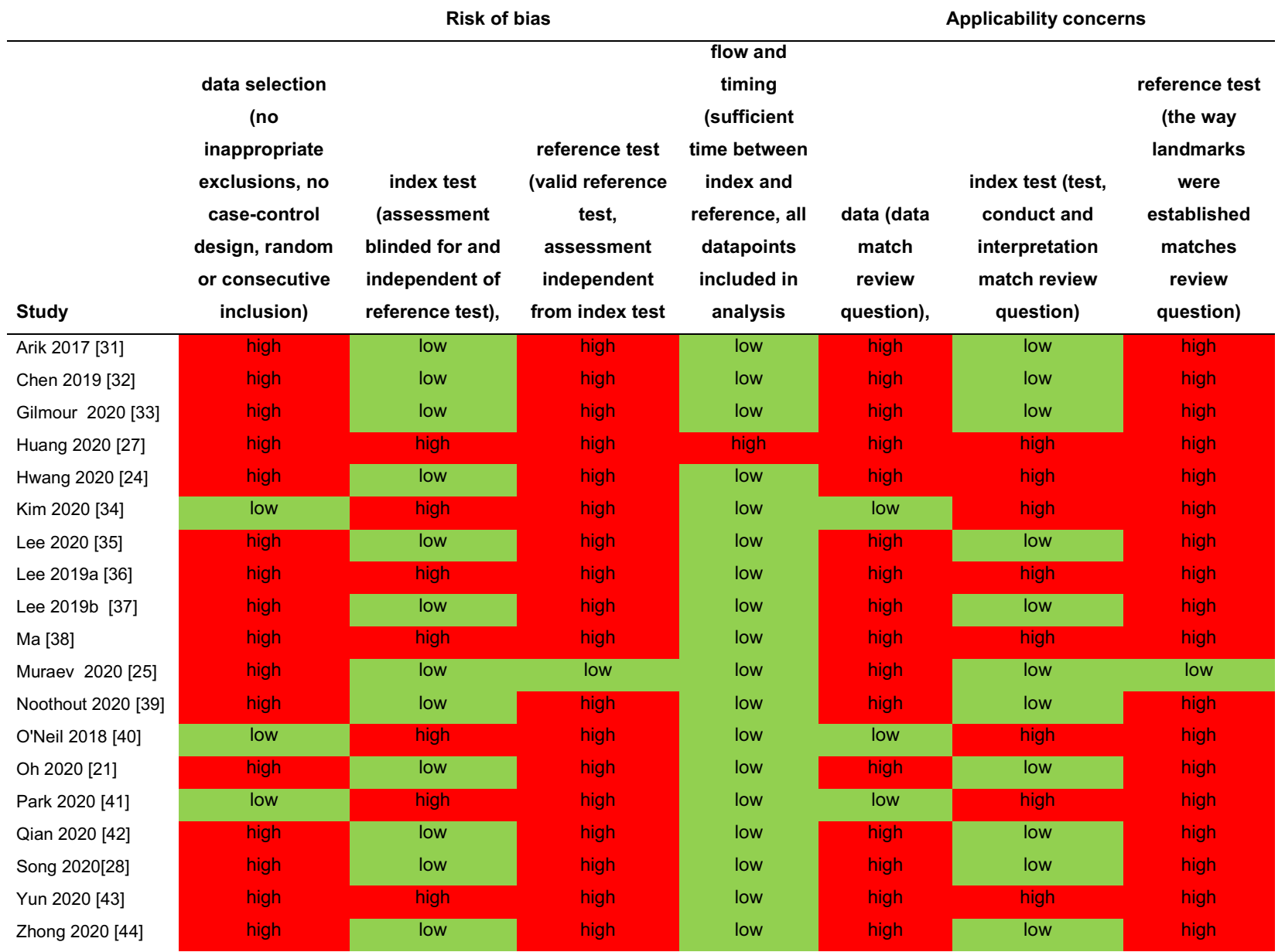

A number of our findings need to be discussed. First, the reported mean deviations were rather consistent across studies; the detected heterogeneity in our first meta-analysis was mainly due to differences between 2-D and 3-D imagery. The proportion of landmarks detected within the 2-mm threshold varied more markedly, with one study showing only $62 \%$ of landmarks achieving that threshold. When assessing which specific landmarks were prone to not being detected correctly, the porion, subspinale, gonion, articulare, and the anterior nasal spine were most often found to show larger deviations. However, these findings were not necessarily consistent across studies, and for these landmarks, DL did not necessarily perform worse than clinicians. Overall, and based on the two studies which evaluated human comparator groups against DL, we conclude that DL performs similar as regular clinicians [24] or even superior to inexperienced ones [30]. Generally, it is difficult to compare mean deviations between studies, as they largely depend on the test dataset: Even in the widely used and publicly available IEEE dataset, both the clinicians but also DL consistently performed worse on the test dataset 2 (containing 100 images) than dataset 1 (containing 150 images). Moreover, DL may not exceed experts' accuracy but may obviously assist landmark detection for regular or experienced examiners. Training models on larger datasets may eventually help to even be as or more accurate than experts [42].

Second, data on 3-D imagery were sparse; only four studies employed DL for this purpose. A recent systematic review compared DL with knowledge-, atlas- and shallow-learningbased methods for 3-D landmark detection and concluded that DL was most accurate [13]. Given the paucity in data, however, it is difficult to strongly endorse DL for 3-D landmark detection at present. Generally, it should be considered that CBCT-based assessments will not be the rule for many orthodontic patients, but rather the exception, for example, when planning orthognatic surgery [9].

Third, we did not identify significant changes in accuracy in studies published in different years. One may expect more recent studies to show higher accuracies, as larger datasets, more powerful hardware, and more effective DL architectures might be available. Regarding the datasets, this was obviously not the case; the usage of the IEEE 2015 dataset was as common in 


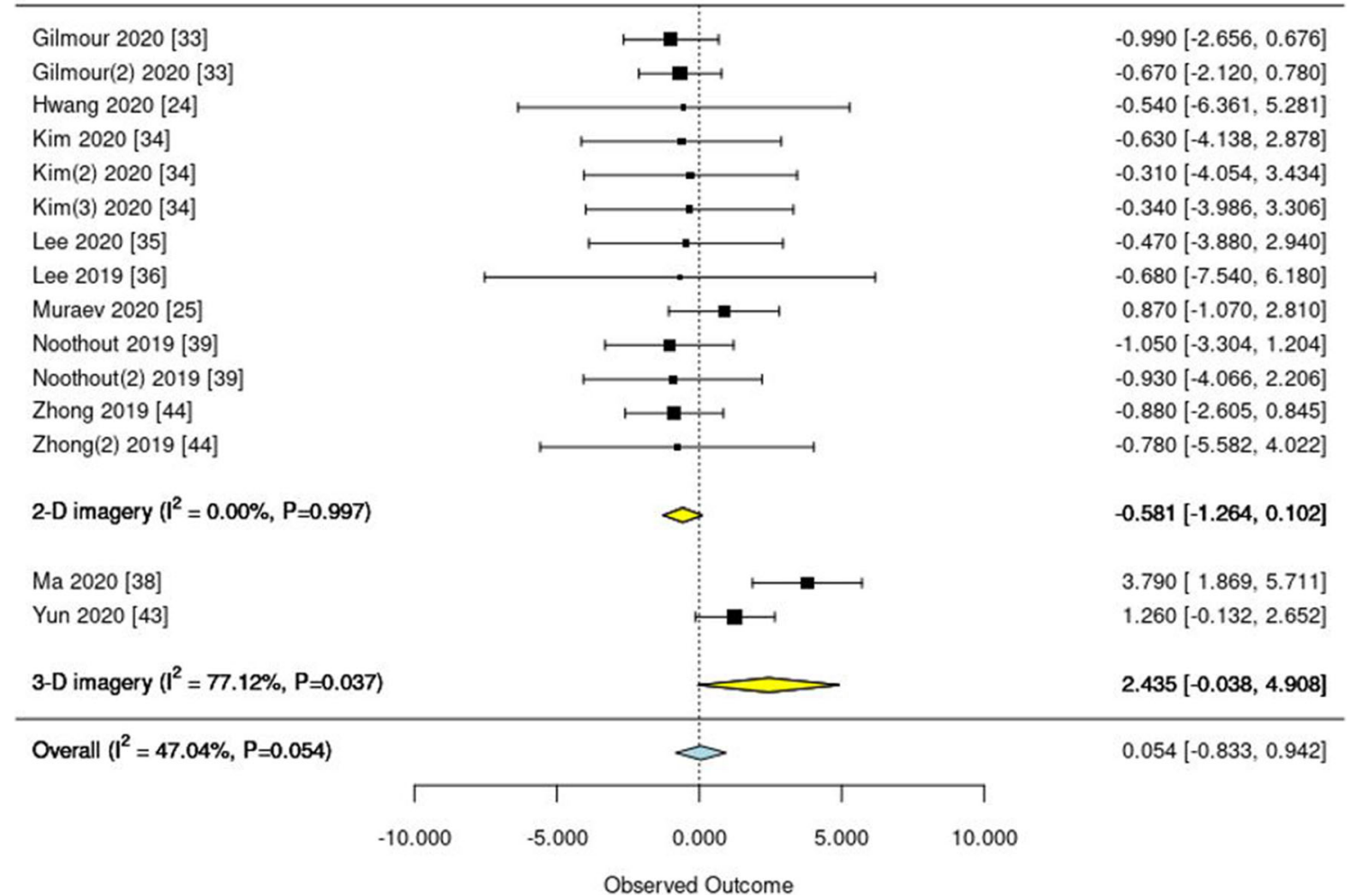

Fig. 2 Forest plot of studies reporting the mean deviation from a 2-mm prediction error threshold. Squares indicate the mean deviation of each single study and lines the $95 \%$ confidence intervals (95\% CI). Yellow and blue diamonds show the pooled subtotal (on 2-D and 3-D imagery) and

2020 as it was in 2017-2019. Moreover, it is likely that more powerful hard- or software can only be limitedly leveraged on datasets containing only a few hundred images like the IEEE 2015 one. Also, we did not identify a consistent evolution of the employed architectures and found only limited benefit of newer architectures (there was only one study on this issue, and this study found the accuracies of LeNet-5 and ResNet50 on the same 3-D test data to be similar) [23].

Fourth, the relevance of the test dataset (as discussed) was confirmed. The consistent difference in accuracy on the two test datasets of the IEEE 2015 challenge has been mentioned; an even more dramatic drop in accuracy was found when models were tested on a fully external dataset [36]. It is commendable that given the IEEE dataset composition, many studies had two test datasets. However, as all studies tested in this same dataset (and most also trained on this dataset), we likely have high comparability but limited generalizability. Future studies should aim to test DL models on broad data, demonstrating robustness and generalizability.

This review and the included studies have a number of limitations. First, we focused on DL for landmark detection; a comparison against other (semi-)automated landmarking methods has not been conducted. Second, we had to exclude a number of studies, e.g., those using DL for predicting skeletal overall estimates, respectively. I-square and the P value indicate heterogeneity. Studies are ordered according to year; if multiple test datasets were employed in the same study, the second or third is indicated accordingly (e.g., Noothout 2019 (2))

anomalies (i.e., skipping landmark detection and analysis) or those which were unavailable in full text, likely losing some valuable data. Third, the included studies suffered from a range of risks of bias. Data selection yielded small and possibly nonrepresentative populations; the majority of studies employed the same dataset (meaning that all these studies can show their DL model to work on exactly this single test dataset, not on data from other populations). Regarding data representativeness, the overall evidence was highly limited; especially for 3-D imagery, scans in the test dataset usually stemmed from only few patients. The reference test (i.e., how the ground truth was established) was only sparsely described; in many studies, it was not exactly clear how the labels of one or more human annotator(s) eventually resulted in the training and test dataset. Some studies used only one expert as reference test, a decision which may be criticized given the wide variability in experts' landmarking, as discussed. Any DL model trained on such dataset will be only as good as this single expert. Fourth, and as discussed, only few studies tested the developed DL models on truly independent datasets, e.g., from different centers, populations, or image generators, contributing to the limitations in generalizability. Fifth, it must be kept in mind that a location error of $<2.0 \mathrm{~mm}$ may be acceptable for some, but not all landmarks: For example, the location error of the $\mathrm{A}$ and $\mathrm{B}$ point 


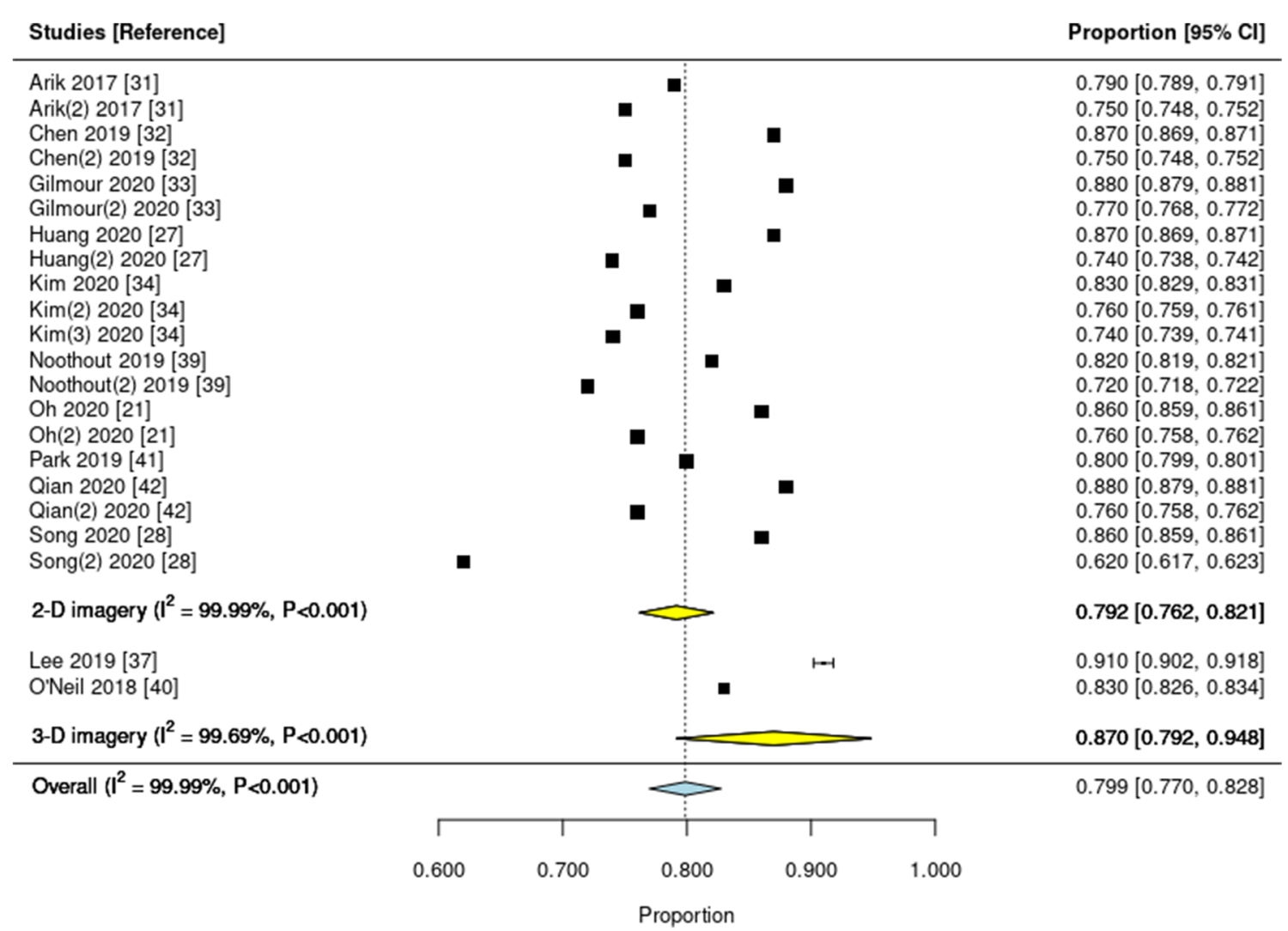

Fig. 3 Forest plot of studies reporting the proportion of landmarks correctly predicted within a $2-\mathrm{mm}$ prediction error threshold from the reference. Squares indicate the mean proportion found in each single study and lines the $95 \%$ confidence intervals (95\% CI). Yellow and blue diamonds show the pooled subtotal (on 2-D and 3-D imagery) and overall

is usually large in the vertical and small in the horizontal plane, the latter being the important direction for determining the sagittal jaw relationship $[8,41]$. In this direction and on these points, location errors of $1.9 \mathrm{~mm}$ would be considered inacceptable $[8,43]$ It should be also kept in mind that socalled stable reference structures are subject to variation, with dental experts remaining needed to critically assess AI findings [44] Last, the studies mainly employed accuracy estimates (this was partially the result of our inclusion criteria), while different outcome measures (deviations in $\mathrm{mm}$, pixels, or proportions) were employed, which are not necessarily comparable. Further outcomes with relevance to clinicians, patients, or other stakeholders (like the impact of using a DL tool in clinical routine on diagnostic and treatment processes, their efficacy, safety, or efficiency) were not reported. Future studies should consider including a wider outcome set and aim to test DL applications comprehensively in other study designs and settings (e.g., observational studies in clinical care, randomized controlled trials). Also, it should be considered that the requirements toward AI-based cephalometric analyses may differ according to the resulting treatment decisions: Deviations acceptable when planning aligner treatments in Class I patients may be intolerable when planning surgical interventions, for instance. estimates, respectively. I-square and the $\mathrm{P}$ value indicate heterogeneity. Studies are ordered according to year; if multiple test datasets were employed in the same study, the second or third is indicated accordingly (e.g., Arik 2017 (2))

\section{Conclusion}

DL shows relatively high accuracy for detecting landmarks on cephalometric imagery. The majority of studies focused on 2-D imagery; data on 3-D imagery are sparse, but promising. There is heterogeneity in detection accuracy between landmarks, and it remains unclear if clinicians are similar, more or less accurate than DL for different landmarks. The overall evidence, while by large consistent, is of limited generalizability and robustness, and the true value of using DL in clinical care needs to be demonstrated.

Supplementary Information The online version contains supplementary material available at https://doi.org/10.1007/s00784-021-03990-w.

Funding Open Access funding enabled and organized by Projekt DEAL.

\section{Declarations}

Ethics approval Not needed.

Consent to participate Not needed.

Conflict of interest FS and JK are co-founders of a startup on deep learning-based dental image analysis. The planning, conduct, and 
reporting of this study was fully independent. This study was self-funded by the authors and their institutions.

Supplementary Information The online version contains supplementary material available at https://doi.org/10.1007/s00784-021-03990-w.

Open Access This article is licensed under a Creative Commons Attribution 4.0 International License, which permits use, sharing, adaptation, distribution and reproduction in any medium or format, as long as you give appropriate credit to the original author(s) and the source, provide a link to the Creative Commons licence, and indicate if changes were made. The images or other third party material in this article are included in the article's Creative Commons licence, unless indicated otherwise in a credit line to the material. If material is not included in the article's Creative Commons licence and your intended use is not permitted by statutory regulation or exceeds the permitted use, you will need to obtain permission directly from the copyright holder. To view a copy of this licence, visit http://creativecommons.org/licenses/by/4.0/

Open Access This article is licensed under a Creative Commons Attribution 4.0 International License, which permits use, sharing, adaptation, distribution and reproduction in any medium or format, as long as you give appropriate credit to the original author(s) and the source, provide a link to the Creative Commons licence, and indicate if changes were made. The images or other third party material in this article are included in the article's Creative Commons licence, unless indicated otherwise in a credit line to the material. If material is not included in the article's Creative Commons licence and your intended use is not permitted by statutory regulation or exceeds the permitted use, you will need to obtain permission directly from the copyright holder. To view a copy of this licence, visit http://creativecommons.org/licenses/by/4.0/.

\section{References}

1. LeCun Y, Bengio Y, Hinton G (2015) Deep learning. Nature 521(7553):436-444

2. Marcus G, Deep learning: a critical appraisal, arXiv.org https:// arxiv.org/abs/1801.00631 (2018)

3. Schwendicke F, Samek W, Krois J (2020) Artificial intelligence in dentistry: chances and challenges. J Dent Res 99(7):769-774

4. Schwendicke F, Golla T, Dreher M, Krois J, Convolutional neural networks for dental image diagnostics: a scoping review, Journal of dentistry (2019) 103226

5. Walsh T (2018) Fuzzy gold standards: approaches to handling an imperfect reference standard. J Dent 74(Suppl 1):S47-s49

6. Schwendicke F, Singh T, Lee JH, Gaudin R, Chaurasia A, Wiegand $\mathrm{T}$, Uribe $\mathrm{S}$, Krois J, Artificial intelligence in dental research: checklist for authors, reviewers, readers, Journal of dentistry (2021) 103610 .

7. Cook AH, Sellke TA, BeGole EA (1994) The variability and reliability of two maxillary and mandibular superimposition techniques. Part II, American journal of orthodontics and dentofacial orthopedics : official publication of the American Association of Orthodontists, its constituent societies, and the American Board of Orthodontics 106(5):463-471

8. Miethke R-R (1995) Possibilities and limitations of various cephalometric variables and analyses. Mosby-Wolfe, St. Louis

9. American Academy of Oral and Maxillofacial Radiology (2013) Clinical recommendations regarding use of cone beam computed tomography in orthodontics. [corrected]. Position statement by the American Academy of Oral and Maxillofacial Radiology. Oral Surg Oral Med Oral Pathol Oral Radiol 116(2):238-257
10. Helal NM, Basri OA, Baeshen HA (2019) Significance of cephalometric radiograph in orthodontic treatment plan decision. J Contemp Dent Pract 20(7):789-793

11. Lagravère MO, Low C, Flores-Mir C, Chung R, Carey JP, Heo G, Major PW (2010) Intraexaminer and interexaminer reliabilities of landmark identification on digitized lateral cephalograms and formatted 3-dimensional cone-beam computerized tomography images. American journal of orthodontics and dentofacial orthopedics : official publication of the American Association of Orthodontists, its constituent societies, and the American Board of Orthodontics 137(5):598-604

12. Hassan B, Nijkamp P, Verheij H, Tairie J, Vink C, van der Stelt P, van Beek H (2013) Precision of identifying cephalometric landmarks with cone beam computed tomography in vivo. Eur $\mathrm{J}$ Orthod 35(1):38-44

13. Dot G, Rafflenbeul F, Arbotto M, Gajny L, Rouch P, Schouman T (2020) Accuracy and reliability of automatic three-dimensional cephalometric landmarking. Int J Oral Maxillofac Surg 49(10): 1367-1378

14. Moher D, Liberati A, Tetzlaff J, Preferred reporting items for systematic reviews and meta-analyses: the PRISMA statement, Ann Intern Med 151 (2009)

15. McKinney M (2011) arXiv.org. Ref Rev 25(7):35-36

16. Whiting P, Rutjes A, Westwood M, Mallett S, Deeks J, Reitsma J, Leeflang M, Sterne J, Bossuyt P, QUADAS-2 Group (2011) QUADAS-2: a revised tool for the quality assessment of diagnostic accuracy studies. Ann Intern Med 155(8):529-536

17. Higgins JPT, Thompson SG (2002) Quantifying heterogeneity in a meta-analysis. Stat Med 21(11):1539-1558

18. Viechtbauer W, Conducting meta-analyses in $\mathrm{R}$ with the metafor package, Journal of Statistical Software; Vol 1, Issue 3 (2010) (2010)

19. Wallace BC, Dahabreh IJ, Trikalinos TA, Lau J, Trow P, Schmid CH (2012) OpenMetaAnalyst: closing the gap between methodologists and end-users: R as a computational back-end. J Stat Softw 49(5)

20. Ark SÖ, Ibragimov B, Xing L (2017) Fully automated quantitative cephalometry using convolutional neural networks. J Med Imaging (Bellingham) 4(1):014501-014501

21. Chen R, Ma Y, Chen N, Lee D, Wang W, Cephalometric landmark detection by attentive feature pyramid fusion and regression-voting, MICCAI 2019 arXiv:1908.08841 (2019)

22. Gilmour L, Ray N, Locating cephalometric x-ray landmarks with foveated pyramid attention, MIDL 2020 arXiv:2008.04428v1 (2020)

23. Huang Y, Fan F, Syben C, Roser P, Mill L, Maier A, Cephalogram synthesis and landmark detection in dental cone-beam CT systems, arXiv:2009.04420 (2020)

24. Hwang HW, Park JH, Moon JH, Yu Y, Kim H, Her SB, Srinivasan G, Aljanabi MNA, Donatelli RE, Lee SJ (2020) Automated identification of cephalometric landmarks: part 2- might it be better than human? The Angle orthodontist 90(1):69-76

25. Kim H, Shim E, Park J, Kim YJ, Lee U, Kim Y (2020) Web-based fully automated cephalometric analysis by deep learning. Comput Methods Prog Biomed 194:105513

26. Lee JH, Yu HJ, Kim MJ, Kim JW, Choi J (2020) Automated cephalometric landmark detection with confidence regions using Bayesian convolutional neural networks. BMC oral health 20(1): 270

27. Lee C, Tanikawa C, Lim J-Y, Yamashiro T, Deep learning based cephalometric landmark identification using landmark-dependent multi-scale patches, arXiv:1906.02961v1 (2019)

28. Lee SM, Kim HP, Jeon K, Lee SH, Seo JK (2019) Automatic 3D cephalometric annotation system using shadowed 2D image-based machine learning. Phys Med Biol 64(5):055002 
29. Ma Q, Kobayashi E, Fan B, Nakagawa K, Sakuma I, Masamune K, Suenaga H (2020) Automatic 3D landmarking model using patchbased deep neural networks for CT image of oral and maxillofacial surgery, The international journal of medical robotics + computer assisted surgery. MRCAS 16(3):e2093

30. Muraev AA, Tsai P, Kibardin I, Oborotistov N, Shirayeva T, Ivanov S, Ivanov S, Guseynov N, Aleshina O, Bosykh Y, Safyanova E, Andreischev A, Rudoman S, Dolgalev A, Matyuta M, Karagodsky V, Tuturov N (2020) Frontal cephalometric landmarking: humans vs artificial neural networks. Int J Comput Dent 23(2): 139-148

31. Noothout JMH, De Vos BD, Wolterink JM, Postma EM, Smeets PAM, Takx RAP, Leiner T, Viergever MA, Isgum I (2020) Deep learning-based regression and classification for automatic landmark localization in medical images. IEEE Trans Med Imaging 39(12): 4011-4022

32. O'Neil AQ, Kascenas A, Henry J, Wyeth D, Shepherd M, Beveridge E, Clunie L, Sansom C, Šeduikyte E, Muir K, Poole I (2019) Attaining human-level performance with atlas location autocontext for anatomical landmark detection in 3D CT data. In: Leal-Taixé L, Roth S (eds) Computer Vision - ECCV 2018 Workshops. Springer International Publishing, Cham, pp 470-484

33. Oh K, Oh IS, Le TVN, Lee DW, Deep anatomical context feature learning for cephalometric landmark detection, IEEE journal of biomedical and health informatics Pp (2020)

34. Park JH, Hwang HW, Moon JH, Yu Y, Kim H, Her SB, Srinivasan G, Aljanabi MNA, Donatelli RE, Lee SJ (2019) Automated identification of cephalometric landmarks: part 1-comparisons between the latest deep-learning methods YOLOV3 and SSD. The Angle orthodontist 89(6):903-909

35. Qian J, Luo W, Cheng M, Tao Y, Lin J, Lin H (2020) CephaNN: a multi-head attention network for cephalometric landmark detection. IEEE Access 8:112633-112641
36. Song Y, Qiao X, Iwamoto Y, Chen Y-w (2020) Automatic cephalometric landmark detection on X-ray images using a deep-learning method. Appl Sci 10(7):2547

37. Yun HS, Jang TJ, Lee SM, Lee SH, Seo JK (2020) Learning-based local-to-global landmark annotation for automatic 3D cephalometry. Phys Med Biol 65(8):085018

38. Zhong Z, Li J, Zhang Z, Jiao Z, Gao X, An attention-guided deep regression model for landmark detection in cephalograms, arXiv: 1906.07549 (2019)

39. Wang CW, Huang CT, Hsieh MC, Li CH, Chang SW, Li WC, Vandaele R, Marée R, Jodogne S, Geurts P, Chen C, Zheng G, Chu C, Mirzaalian H, Hamarneh G, Vrtovec T, Ibragimov B (2015) Evaluation and comparison of anatomical landmark detection methods for cephalometric x-ray images: a grand challenge. IEEE Trans Med Imaging 34(9):1890-1900

40. Björk A (1969) Prediction of mandibular growth rotation. Am J Orthod 55(6):585-599

41. Baumrind S, Frantz RC (1971) The reliability of head film measurements. 1. Landmark identification. Am J Orthod 60(2):111127

42. Samala RK, Chan HP, Hadjiiski L, Helvie MA, Richter CD, Cha KH (2019) Breast cancer diagnosis in digital breast tomosynthesis: effects of training sample size on multi-stage transfer learning using deep neural nets. IEEE Trans Med Imaging 38(3):686-696

43. Steiner CC (1960) The use of cephalometrics as an aid to planning and assessing orthodontic treatment: report of a case. Am J Orthod 46(10):721-735

44. Baumrind S, Miller D, Molthen R (1976) The reliability of head film measurements: 3. Tracing superimposition, American Journal of Orthodontics 70(6):617-644

Publisher's note Springer Nature remains neutral with regard to jurisdictional claims in published maps and institutional affiliations. 